\title{
Optimal Allocation of Unified Power Flow Controller via Bat Algorithm
}

Sadhvi Ajit Varagiri

University of Michigan Dearborn, USA

sadhvi.a.v@gmail.com

\begin{abstract}
The Unified Power Flow Controller (UPFC) is one of the most promising Flexible AC Transmission Systems (FACTS) devices for the load flow control". Therefore, optimizing the position and size of UPFCs is a significant concern for raising the loading capability of the system. So far, several optimization approaches were introduced for resolving the issues in UPFC. Here, this work intends to establish a Bat Algorithm (BA) model for optimal sizing and positioning of UPFC to attain Optimal Power Flow (OPF). The deployed model is the improved edition of the conventional BA algorithm. Moreover, the objective specified for resolving the placement issue comprises of the reduction of cost and power loss. At last, the supremacy of developed model is evaluated over conventional models in terms of power loss. From the analysis, the performance of the implemented BA is $5 \%$ and $2.06 \%$ better than the compared ABC and GSA models.
\end{abstract}

Keywords: UPFC; FACTS; Bat Algorithm; Optimal flow Control; Power Quality.

\begin{tabular}{ll} 
Nomenclature & \\
\hline Abbreviation & Description \\
\hline ABC & Artificial Bee Colony \\
APLFs & Active Power Loop Flows \\
CPS-SPWM & Carrier Phase Shifted Sinusoidal Pulse Width Modulation \\
DG & Distribution Generation \\
DE & Differential Evolutionary \\
EP & Evolutionary Programming \\
FACTS & Flexible AC Transmission Systems \\
GSA & Gravitational Search Algorithm \\
GU & Generation Unit \\
GA & Genetic Algorithm \\
HBC & Half-Bridge Converter \\
HICA-PS & Hybrid Imperialist Competitive Algorithm-Pattern Search \\
ITLBO & Improved Teaching Learning Based Optimization \\
IPM & Interior Point Method \\
MMC & Modular Multilevel Converter \\
OPF & Optimal Power Flow \\
OKHA & Oppositional Krill Herd Algorithm \\
PQ & Power Quality \\
RA & Resul Accuracy \\
SCA & Sine Cosine Algorithm \\
TCR & Thyristor Controlled Reactor \\
UPFC & Unified Power Flow Controller \\
\hline
\end{tabular}

\section{Introduction}

Nowadays, acceptable level of PQ is a primary challenge in a DG system for various nonlinear loads. "PQ $[1,2,3]$ is termed as the quality of electric power distributed to customers, which includes generation, distribution and transmission systems". Amongst all, the distribution system is a primary feature that holds a very important role in power consumption. The distribution system [6, 7] divide up the electrical 
and power utilities to customers. The intention of an optimal power system is to distribute electricity to its customers in a reliable and well-organized manner [8,9,10].

The power factor, stability of service, harmonics reliability and voltage distortions are certain computational indicators that portray the effectiveness of the intellect PQ system [11] [12]. Nevertheless, the issues such as impedance and resonance create the system performance more multifaceted and defective $[1,13,14]$. Accordingly, for prevailing over these problems, the FACTS devices namely; TCR, UPFC [1,2] are employed, which results in superior electrical power quality. It also offers varied harmonic orders and recompenses for reactive power [3,5]. In literature there were 3 techniques for reducing voltage sags. They are "UPQC-Q, UPQC-P and minimum VA loading of UPQC". In the aforesaid techniques, the lessening of voltage sag with phase jumps is for the positive sequence voltage. Usually, voltage sag is characterized by sag depth, phase jump and duration of sag. But, most of the voltage sags are deep and long duration sag which has negative impact when compared to shallow and short duration sags on sensitive loads. As sag severity increases, the UPQC require more amount of VA to mitigate.

A number of FACTS controllers were designed for regulating the power system at normal and contingency conditions. Among such FACT devices, UPFC exhibits the excellent characteristics that assist the power system to function securely and reliably [3]. UPFC [7] is an adjustable device that plays a main role in controlling the entire power system. The principal task of UPFC [6] is to manage the OPF of load in the electrical system. In addition, the optimal allocation of UPFC [5] is essential to achieve better system performance and cost-efficacy. There are numerous techniques in the literature for resolving the issues on UPFC allocation. "Sensitivity-based analysis and optimization and index calculation methods" are certain general techniques exploited for allocating UPFC. Some of the familiar heuristic approaches for determining the optimal allotment of UPFC include DE, GA, EP model, and IPM algorithms.

The arrangement of the paper is: Section 2 portrays the review. Section 3 gives a short interpretation on modelling of UPFC system; section 4 defines the BA oriented optimization for optimal flow control of UPFC. Section V portrays the outcomes and the paper is concluded by section 5 .

\section{Literature review}

\subsection{Related Works}

In 2017, Ravindra et al. [1] have developed an ITLBO model for examining the security of system under contingency conditions. For enhancing the security, the UPFC device was positioned optimally in the system. For optimal positioning, the power injection model and optimal location detection models were introduced. In the end, the introduced method was investigated in terms of security and optimal outcomes were attained.

In 2019, Vural and Emile [2] adopted a comprehensive model of a MMC oriented UPFC. Accordingly, a high switching frequency system known as CPS-SPWM was modelled for controlling the capacitor voltage in the sub-modules of HBC. The suggested scheme was further evaluated and the outcomes have revealed the benefits of the developed method over the other compared methods through case studies.

In 2016, Dutta et al. [3] have adopted a technique for attaining optimal steady-state system depending on OKHA method. This work has examined the impact of UPFC allotment by means of steady-state analysis and it also established the capability of UPFC in balancing the reactive and active flows of power. For confirming the efficacy of the developed technique, enhancement of voltage and reduction of real power losses were taken into concern and their outcomes were found to be proficient and practicable.

In 2016, Majid et al. [4] have introduced a load shedding coordination design for preventing the voltage collapses. In addition, the contingency state was evaluated via HICA-PS model that holds a most important role in tuning the ICA outcomes. For representing the efficacy of the offered scheme, it was assessed over the traditional schemes concerning voltage collapse. Here, the modelled system primarily focused on the reduction of voltage fall down, load shedding and reliability of energy distribution.

In 2020, Li et al. [5] have examined the active power levels of UPFC for eliminating the APLFs. The sensitivity amongst the active power of UPFC was resolved for achieving zero active power flow. In addition, the coordination amongst the converters was determined for portraying the controllable ranges for varied voltage settings. The coordination of numerous UPFCs was presented for deciding the active power of diverse UPFCs. Eventually, the outcomes have established the improvement of the adopted scheme in terms of its capability. 


\section{A Short Interpretation On Modeling Of UPFC System}

The modelling of UPFC system is demonstrated in Fig. 1, wherein the generator Ge is connected with the buses $\mathrm{n}$ and $\mathrm{m}$. Accordingly, the converters are connected by the transformer. It entails the converter and load impedance pointed out by $\mathrm{Z}_{\mathrm{H}}$ and $\mathrm{Z}_{\mathrm{L}}$ in that order. The converters are associated with voltage $\mathrm{V}_{\mathrm{dc}}$ capacity with DC link capacitor $\mathrm{C}_{\mathrm{dc}}$. It is incorporated with the UPFC power flow formulations [10], which is crucial for resolving the inequality and equality issues. It might occur due to the outage of generators available in the power system, as the requirements should be satisfied constantly. The inequality and equality constraints are explained in brief in the below part.

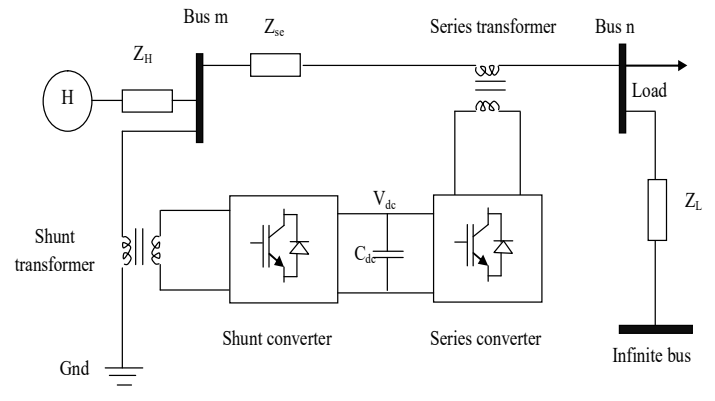

Fig. 1. Modelling of UPFC

Equality constraints: The foremost contribution of the power system relies on the achievement of total demand of utility [11]. Here, Ge have to gratify the entire requirement of the customers and it is believed to satisfy the loss of power in transmission lines. This is recognized as the equality power balance state or constraints of the power system. The desired equality constraints are described as per Eq. (1), $P_{\mathrm{H}}^{\mathrm{a}}$ points out the power produced in $\mathrm{a}^{\text {th }}$ bus, $\mathrm{P}_{\mathrm{B}}$ points out the demand, $\mathrm{Q}_{\mathrm{L}}^{\mathrm{b}}$ and $\mathrm{P}_{\mathrm{L}}^{\mathrm{b}}$ points out the reactive and real power losses of $b^{\text {th }}$ bus that are computed as per Eq. (2) and Eq. (3).

$$
\sum_{\mathrm{a}=1}^{\mathrm{M}_{\mathrm{H}}} \mathrm{P}_{\mathrm{H}}^{\mathrm{a}}=\mathrm{P}_{\mathrm{B}}+\sum_{\mathrm{b}=1}^{\mathrm{M}_{\mathrm{H}}}\left(\mathrm{P}_{\mathrm{L}}^{\mathrm{b}}+\mathrm{b} \mathrm{Q}_{\mathrm{L}}^{\mathrm{b}}\right)
$$

In Eq. (1),

$$
\begin{aligned}
& \mathrm{Q}_{\mathrm{L}}^{\mathrm{b}}=\left|\mathrm{V}_{\mathrm{a}}\right|\left|\mathrm{V}_{\mathrm{b}}\right|\left|\mathrm{X}_{\mathrm{ab}}\right| \sum_{\mathrm{n}=1}^{\mathrm{M}} \sin \left(\delta_{\mathrm{ab}}-\alpha_{\mathrm{a}}-\alpha_{\mathrm{b}}\right) \\
& \mathrm{P}_{\mathrm{L}}^{\mathrm{b}}=\left|\mathrm{V}_{\mathrm{a}}\right|\left|\mathrm{V}_{\mathrm{b}}\right|\left|\mathrm{X}_{\mathrm{ab}}\right| \sum_{\mathrm{n}=1}^{\mathrm{M}} \cos \left(\delta_{\mathrm{ab}}-\alpha_{\mathrm{a}}-\alpha_{\mathrm{b}}\right)
\end{aligned}
$$

In Eq. (2) and Eq. (3), $\mathrm{V}_{\mathrm{b}}$ and $\mathrm{V}_{\mathrm{a}}$ points out the voltage of the buses $\mathrm{b}$ and $\mathrm{a}, \mathrm{X}_{\mathrm{ab}}$ indicate the matrix of bus admittance, $\delta_{a b}$ stand for the angle amongst buses a and b, $\alpha_{b}$ as well as $\alpha_{a}$ signify the load angles of $\mathrm{b}$ and $\mathrm{a}$.

Inequality constraints: The inequality parameters specifically, reactive and real power flows and voltage are affected due to the issues on GU [11]. The dynamic stability of the power system chiefly focuses on the stability of voltage in every node. For a stable flow of power, the voltage of every bus has to lie among 0.95-1.05 pu. The difference in voltage is pointed out by Eq. (4), wherein $\mathrm{V}_{\mathrm{a}}^{\mathrm{k}}$ is evaluated as per Eq. (5).

$$
\begin{aligned}
& \Delta \mathrm{V}_{\mathrm{i}}=\frac{1}{\sqrt{\mathrm{l}}} \sqrt{\sum_{\mathrm{a}=1}^{\mathrm{l}}\left(\mathrm{V}_{\mathrm{a}}^{\mathrm{k}}\right)^{2}} \\
& \mathrm{~V}_{\mathrm{a}}^{\mathrm{k}}=\mathrm{V}_{\text {slack }}-\sum_{\mathrm{a}=1}^{\mathrm{n}} \mathrm{Z}_{\mathrm{a}}\left(\frac{\mathrm{P}_{\mathrm{a}}-\mathrm{bQ}_{\mathrm{a}}}{\mathrm{V}_{\mathrm{a}}}\right)
\end{aligned}
$$

In Eq. (4), $V_{\text {slack }}$ signify the slack bus voltage, $\Delta \mathrm{V}_{\mathrm{a}}$ point out the voltage stability index of $\mathrm{a}^{\text {th }}$ bus, wherein $\mathrm{a}=1,2, \ldots . \mathrm{n}, \mathrm{Z}_{\mathrm{a}}$ specify the impedance of $\mathrm{a}^{\text {th }}$ bus, $\mathrm{Q}_{\mathrm{a}}$ and $\mathrm{P}_{\mathrm{a}}$ specify reactive and real powers of bus $a$ and the count of nodes is specified by b. The bus voltage relies amid the bounds, i.e. $\mathrm{V}_{\mathrm{a}}^{\min } \leq \mathrm{V}_{\mathrm{a}} \leq \mathrm{V}_{\mathrm{a}}^{\max }$ : The reactive and real powers of the specific bus are computed as in Eq. (6) and Eq. 
(7), where $M_{D}$ indicate the total count of buses $\alpha_{a b}$ symbolize the angle between buses a and $b$ in that order, $\mathrm{D}_{\mathrm{ab}}$ and $\mathrm{H}_{\mathrm{ab}}$ stand for the susceptance and conductance values, in that order.

$$
\begin{aligned}
& \mathrm{Q}_{\mathrm{a}}=\left|\mathrm{V}_{\mathrm{a}}\right|\left|\mathrm{V}_{\mathrm{b}}\right| \sum_{\mathrm{n}=1}^{\mathrm{M}_{\mathrm{D}}}\left(\mathrm{H}_{\mathrm{ab}} \sin \alpha_{\mathrm{ab}}-\mathrm{D}_{\mathrm{ab}} \cos \alpha_{\mathrm{ab}}\right) \\
& \mathrm{P}_{\mathrm{a}}=\left|\mathrm{V}_{\mathrm{a}}\right|\left|\mathrm{V}_{\mathrm{b}}\right| \sum_{\mathrm{n}=1}^{\mathrm{M}_{\mathrm{D}}}\left(\mathrm{H}_{\mathrm{ab}} \cos \alpha_{\mathrm{ab}}+\mathrm{D}_{\mathrm{ab}} \sin \alpha_{\mathrm{ab}}\right)
\end{aligned}
$$

\section{BA Oriented Optimization For Optimal Allocation Of UPFC}

\subsection{Solution Encoding and Objective Function}

The implemented approach controls the optimal flow control in UPFC by optimizing the angle, compensation, tap setting and location. For optimization, a model known as BA is deployed in this work. The input solution provided to the BA model is demonstrated in Fig. 2, wherein, L stand for the location, $\mathrm{C}$ correspond to the compensation and its limits lies between to 0.17, TS point out the tap setting and its limits lies among 0 to 1 and $\alpha$ indicate the angle and it lies amongst 0 to $2 \pi$.

Moreover, a single objective function (OF) is described in this work that concerns on the lessening of cost and power loss as depicted in Eq. (10), where $f_{1}$ and $f_{2}$ are computed as revealed in Eq. (8) and (9). Here PV point out penalty voltage, $\mathrm{u}_{1}$ and $\mathrm{u}_{2}$ holds a value of $0.8, \mathrm{P}_{\mathrm{L}}$ denote the power loss and $\mathrm{C}$ correspond to the cost.

$$
\begin{aligned}
\mathrm{f}_{1} & =\mathrm{u}_{1} * \mathrm{C}+\left(1-\mathrm{u}_{1}\right) * \mathrm{PV} \\
\mathrm{f}_{2} & =\mathrm{u}_{2} * \mathrm{f}_{1}+\left(1-\mathrm{u}_{2}\right) * \mathrm{P}_{\mathrm{L}} \\
\mathrm{OF} & =\min \left(\mathrm{f}_{2}\right)
\end{aligned}
$$

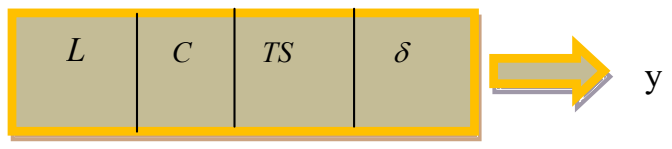

Fig. 2. Solution Encoding

\subsection{BAT Algorithm}

The standard bat algorithm has many advantages, and one of the key advantages is that it can provide very quick convergence at a very initial stage by switching from exploration to exploitation. The significant stages of BA [14] consist of "initialization, evaluation, movement of virtual bats, loudness and pulse rate-based movement and termination".

Initialization: The primary phase is initialization, where the $\mathrm{n}$ bats are chosen arbitrarily and the bat's positions are initialized within search space. Moreover, the factors like iteration count $t$, rate of pulse $r$, loudness $D$, maximal and minimal frequency of $G_{\max }$ and $G_{\min }$, velocity $s_{i}^{t}$, are also initialized in an arbitrary manner. The input image is modelled as revealed in Eq. (8), in which, $y_{i}^{m n}$ signify $i^{\text {th }}$ position of input image with $\mathrm{n}_{\text {columns and }} \mathrm{m}_{\text {rows. }}$.

$$
\mathrm{Y}_{\mathrm{i}}=\left\{\begin{array}{ll}
\mathrm{y}_{\mathrm{i}}^{\mathrm{mn}} & 0 \leq \mathrm{m} \leq \mathrm{q} \\
& 0 \leq \mathrm{n} \leq \mathrm{f}_{\mathrm{n}}
\end{array}\right\}
$$

Evaluation: After initialization process, all bats are computed with the fitness function that focuses on the reduction of loss. If the computed fitness is improved, that value is measured as optimal one $\mathrm{y}_{\mathrm{b}}$.

Movement of bats: After valuation, the position of all maps is updated by means of both frequency and velocity. The formulation of BA is modelled as revealed in Eq. (9), in which $\mathrm{G}_{\mathrm{i}}$ points out the image frequency and $\gamma$ points out the arbitrary integer.

$$
\mathrm{G}_{\mathrm{i}}=\mathrm{G}_{\min }+\left(\mathrm{G}_{\max }-\mathrm{G}_{\min }\right) * \gamma
$$

Consequently, the image velocity is updated using both optimal values of frequency $\mathrm{G}_{\mathrm{i}}$, which are addressed by Eq. (10) and Eq. (11). Furthermore, $\mathrm{y}_{\mathrm{i}}^{\mathrm{t}-1}$ points out the prior iteration, $\mathrm{y}_{\mathrm{b}}$ refers to the best value that is measured as fitness value $\alpha$.

$$
\mathrm{s}_{\mathrm{i}}^{\mathrm{t}}=\mathrm{s}_{\mathrm{i}}^{\mathrm{t}-1}+\mathrm{G}_{\mathrm{i}}\left(\mathrm{y}_{\mathrm{i}}^{\mathrm{t}-1}-\mathrm{y}_{\mathrm{b}}\right)
$$




$$
\mathrm{y}_{\mathrm{i}}^{\mathrm{t}-1}=\mathrm{y}_{\mathrm{i}}^{\mathrm{t}}-\mathrm{s}_{\mathrm{i}}^{\mathrm{t}}
$$

Loudness and pulse rate movement: At this phase, the arbitrary value of $\gamma$ is evaluated with pulse rate denoted by $r$. If the arbitrary value is enhanced than $r$, the novel local solutions are created that depends on the optimal value $\mathrm{y}_{\mathrm{b}}$. Moreover, if the arbitrary value is smaller than the loudness, then arbitrary value is created. Once more, the assessment occurs amongst loudness and arbitrary value. If the arbitrary solution is better than optimal solution, the loudness and pulse rate are updated.

Termination: The aforementioned process is sustained till the entire virtual bats get updated. Following the end of iteration, the output is considered as the optimal one.

\section{Results and discussion}

\subsection{Simulation Procedure}

The developed model for optimal allocation of UPFC using BA was executed in Matlab and the resultants were noticed. The deployed BA model was distinguished over the conventional approaches such as ABC [12] and GSA [13] models. The analysis was carried out on IEEE 30 bus system that included "six generator bus, 21 load bus and 42 transmission lines". In addition, the examination was carried out with respect to accuracy and loss.

\subsection{Analysis On Power Loss}

The power loss (MW) obtained by BA over the existing models for single generator issue is shown in Fig. 4. On observing the outcomes, the BA method has accomplished negligible power loss when evaluated over the traditional models. Here, from Fig. 3, the BA has attained a minimal power loss of 9.5 MW, which is $5 \%$ and $2.06 \%$ enhanced than existing ABC and GSA approaches, when the generator bus number is 2 . This demonstrates the improvement of BA model over the existing schemes.

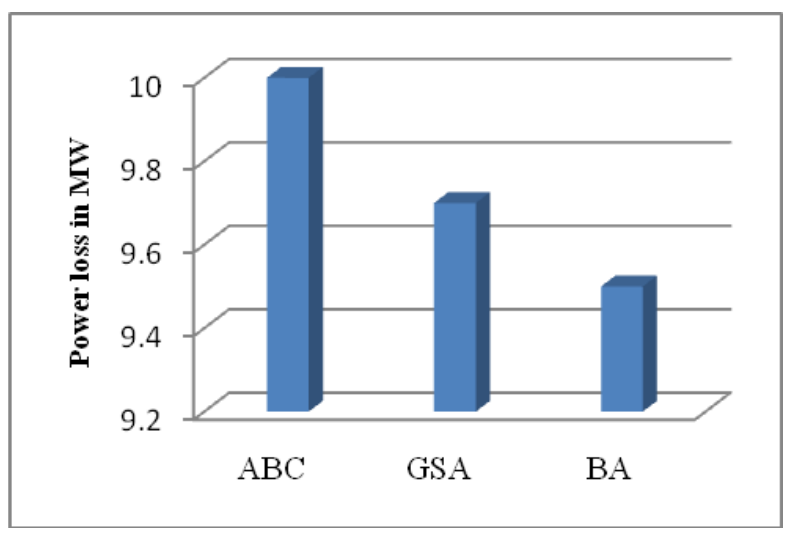

Fig. 3. Power loss analysis of BA over the existing works when the generator bus number $=2$

\subsection{Performance Analysis}

The performance analysis of the BA method over existing approaches is specified in Fig. 3 for IEEE 30 bus system. The investigation was done with regard to $R A$, which is evaluated as $\mathrm{RA}=(\mathrm{BP}-\mathrm{NP}) / \mathrm{NP} \times 100$, wherein $\mathrm{BP}$ signify the best minimal loss of power and $\mathrm{N}$ symbolize the normal loss of power. From the observed outcomes, the RA of BA is found to be high than the distinguished models. That is, the BA is $55 \%$ and $40 \%$ better than the evaluated ABC and GSA models. Therefore, the betterment of the BA method is established from the simulated results. 


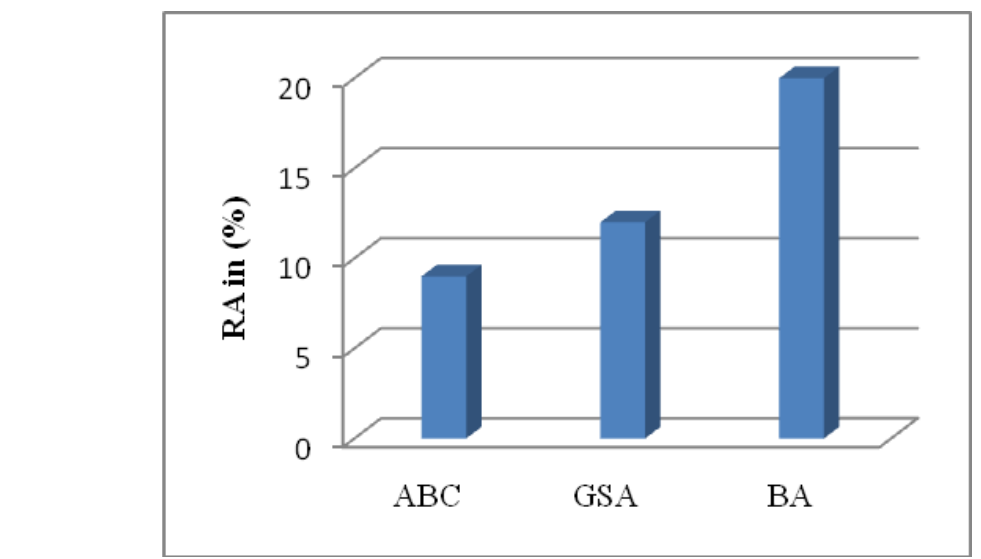

Fig. 4. Performance evaluation of BA over the existing works with respect to $R A$

\subsection{Power Loss Of Double Generator Issue}

Table I illustrate the power loss data attained by means of the BA model for IEEE 30 bus system. The assessment was carried out by altering the generator bus counts from 2 and 6, 2 and 13, 6 and 13, 22 and 27 as well as 13 and 27. From the examination, power loss attained by the BA model at normal condition is 10.809 MW, whereas, during fault condition, 13.74 MW, 14.027 MW, 15.233 MW, 12.031 MW and 13.345 MW are attained for varied generator bus numbers from 2 and 6, 2 and 13, 6 and 13, 22 and 27 and 13 and 27 respectively.

Table 1: Power loss of double generator problem attained by the BA model

\begin{tabular}{|c|c|c|c|c|c|}
\hline \multirow{2}{*}{ Generator bus no } & \multicolumn{2}{|c|}{ Best location } & \multicolumn{3}{|c|}{ Power loss in MW } \\
\hline & From bus & To bus & Normal & During fault & With UPFC \\
\hline 13 and 27 & 10 & 22 & 10.809 & 13.345 & 9.876 \\
\hline 22 and 27 & 12 & 15 & & 12.031 & 9.001 \\
\hline 6 and 13 & 10 & 22 & & 15.233 & 9.768 \\
\hline 2 and 13 & 5 & 7 & & 14.027 & 9.834 \\
\hline 2 and 6 & 12 & 15 & & 13.74 & 9.423 \\
\hline
\end{tabular}

\section{Conclusion}

This paper has exploited BA model for resolving the placement issues of UPFC that aids in enhancing the load flow control. Accordingly, the presented approach intended to resolve two varied problems such as, minimization of UPFC cost and power loss. Consequently, the work primarily focused on the loss and RA attained by the BA over the conventional models. On scrutinizing the outcomes, the RA of the BA was found to be superior to other evaluated models. That is, the BA was $5 \%$ and $2.06 \%$ better than the compared ABC and GSA models. Therefore, the improvement of the presented model was established from the analysis. Thus, the presented model has satisfied the objective function in an optimal way when evaluated over the existing works.

\section{Compliance with Ethical Standards}

Conflicts of interest: Authors declared that they have no conflict of interest.

Human participants: The conducted research follows the ethical standards and the authors ensured that they have not conducted any studies with human participants or animals.

\section{References}

[1] Ravindra, Chintalapudi V. Suresh, S. Sivanagaraju, V. C. Veera Reddy, "Power system security enhancement with unified power flow controller under multi-event contingency conditions", Ain Shams Engineering Journal, vol. 8, no. 1, pp. 9-28, March 2017.

[2] Ahmet Mete Vural, Emile Njodzefon Wirsiy, "Three-phase modular multilevel converter based unified power flow controller", Engineering Science and Technology, an International Journal, In press, corrected proof, Available online 22 May 2019 
[3] Susanta Dutta, Pranabesh Mukhopadhyay, Provas Kumar Roy, Debashis Nandi, "Unified power flow controller based reactive power dispatch using oppositional krill herd algorithm”, International Journal of Electrical Power \& Energy Systems, vol. 80, pp. 10-25, September 2016.

[4] Majid Moazzami, Mohammad Javad Morshed, Afef Fekih, "A new optimal unified power flow controller placement and load shedding coordination approach using the Hybrid Imperialist Competitive AlgorithmPattern Search method for voltage collapse prevention in power system", International Journal of Electrical Power \& Energy Systems, vol. 79, pp. 263-274, July 2016.

[5] Shenghu Li, Ting Wang, Hao Zhang, Lei Wang, Jing Xue., "Sensitivity-based coordination to controllable ranges of UPFCs to avoid active power loop flows", International Journal of Electrical Power \& Energy Systems, vol. 114, January 2020, Article 105383

[6] B. Chen, W. Fei, C. Tian and J. Yuan, "Research on an Improved Hybrid Unified Power Flow Controller," IEEE Transactions on Industry Applications, vol. 54, no. 6, pp. 5649-5660, Nov.-Dec. 2018.

[7] F. Z. Peng, Y. Liu, S. Yang, S. Zhang, D. Gunasekaran and U. Karki, "Transformer-Less Unified Power-Flow Controller Using the Cascade Multilevel Inverter," IEEE Transactions on Power Electronics, vol. 31, no. 8, pp. 5461-5472, Aug. 2016.

[8] R. Thirumalaivasan, Y. Xu and M. Janaki, "Power Control With Z-Source Converter Based Unified Power Flow Controller," IEEE Transactions on Power Electronics, vol. 32, no. 12, pp. 9413-9423, Dec. 2017.

[9] J. Tang et al., "Research on AC transmission line fault ride-through control strategy of MMC-based unified power flow controller", in The Journal of Engineering, vol. 2017, no. 13, pp. 1580-1583, 2017.

[10] Laifa Abdelaziz, Boudour Mohamed, "Optimal placement and parameter settings of unified power flow controller device using a perturbed particle swarm optimization”, IEEE Int Energy Conf Exhibit, 2010:205-10.

[11] B. Vijay Kumar, N. V. Srikanth, "Optimal location and sizing of Unified Power Flow Controller (UPFC) to improve dynamic stability: A hybrid technique”, International Journal of Electrical Power \& Energy Systems, vol. 64, pp. 429-438, January 2015.

[12] Mustafa Servet Kıran, Oğuz Fındık, "A directed artificial bee colony algorithm”, Applied Soft Computing, vol. 26, pp. 454-462, January 2015.

[13] Esmat Rashedi, Hossein Nezamabadi-pour and Saeid Saryazdi, "GSA: A Gravitational Search Algorithm", Information Sciences, vol.179, pp.2232-2248, 2009.

[14] Thomas, Renjith \& Rangachar, M.. (2016). "Integrating GWTM and BAT algorithm for face recognition in lowresolution images. The Imaging Science Journal”. 64. 441-452. 10.1080/13682199.2016.1231990. 